\title{
REVISIÓN DEL GÉNERO Cyclope RISSO, 1826 (GASTROPODA: NASSARIIDAE) EN EL PLIOCENO MEDITERRÁNEO
}

\author{
Carles GILI y Jordi MARTINELL
}

Departamant d'Estratigrafia i Paleontologia, Facultat de Geologia, Universitat de Barcelona. Zona Universitària de Pedralbes, 08071 - Barcelona. e-mail: jordim@natura.geo.ub.es

Gili, C. y Martinell, J. 1999. Revisión del género Cyclope Risso, 1826 (Gastropoda: Nassariidae) en el Plioceno mediterráneo. [Revision of the genus Cyclope Risso, 1826 (Gastropoda: Nassariidae) in the Mediterranean Pliocene.] Revista Española de Paleontología, 14 (1), 147-156. ISSN 0213-6937.

\begin{abstract}
In paleontological literature, Pliocene forms of genus Cyclope are generally considered as $C$. neritea (Linnaeus, 1758), the name corresponding to the Recent Mediterranean species. This is because of the resemblance between the adult forms of both. The members of the genus section the apex of the shell (protoconch and first whorls of the teleoconch) when they reach the adult stage and therefor never exhibit the protoconch. Young Cyclope specimens with the protoconch have been found in the Lower Pliocene sediments of Monteu Roero (Piemonte, NW Italy). The protoconch of fossil specimens is multispiral, whereas the protoconch of the Recent C. neritea is paucispiral. This is sufficient evidence to consider these forms as two different species. The Pliocene species should be named $C$. migliorinii (Bevilacqua, 1928). These are sibling species that differs basically in its protoconch morphology, reflecting different embrionary-larval developments, without any modifications in the young-adult form.

The principal characteristics of the genus are commented on, a detailed description of fossil C. migliorinii and a comparison with Recent $C$. neritea are done. The only apparent consistent difference between the adult forms of both species is the greater overlaping of the last whorl over the former ones in C. migliorinii. Both species also largely coincide in an autoecological context.

The genus Cyclope appeared during the Early Pliocene. C. migliorinii should be considered the first species of the genus. It probably survived until Early Pleistocene (Calabrian), when it was replaced by C. neritea. C. migliorinii inhabited the greater part of the Mediterranean and adjacent areas of the Atlantic, as does $C$. neritea at present.
\end{abstract}

Keywords: Gastropods, Nassariidae, Cyclope, Systematics, Pliocene, Present, Mediterranean.

\section{RESUMEN}

En la literatura paleontológica, las formas del género Cyclope del Plioceno son consideradas, en general, como $C$. neritea (Linnaeus, 1758), nombre de la especie viviente en el Mediterráneo. Esto es debido a la casi total similitud entre los adultos de ambas formas. Los representantes de este género, al llegar al estado adulto, seccionan el ápice de su concha (protoconcha y primeras vueltas de la teleoconcha), por lo cual los adultos nunca presentan la protoconcha. En sedimentos del Plioceno Inferior de Monteu Roero (Piemonte, NO de Italia) se han hallado ejemplares juveniles del género Cyclope que conservan la protoconcha. La protoconcha de los individuos fósiles es multispiral, mientras que la protoconcha de los actuales $C$. neritea es paucispiral. Este dato es suficiente para considerar ambas formas como especies distintas. La especie pliocénica debe designarse como C. migliorinii (Bevilacqua, 1928). Estas son especies gemelas que se diferencian básicamente por la morfología de su protoconcha, lo cual indica deserrollos embrionario-larvario distintos, sin modificaciones en la forma juvenil-adulta.

Se comentan los caracteres más relevantes del género, se hace una descripción detallada de $C$. migliorinii fósil y se realiza la comparación con $C$. neritea actual. La única diferencia que parece constante entre las formas adultas de ambas especies es el mayor recubrimiento de la última vuelta sobre las anteriores en $C$. migliorinii. También desde el punto de vista autoecológico las dos especies coinciden ampliamente.

El género Cyclope apareció en el Plioceno Inferior. C. migliorinii debe considerarse la primera especie de este género. Probablemente perduró hasta el Pleistoceno Inferior (Calabriense), siendo sustituida por $C$. neritea. C. migliorinii se extendió por la mayor parte del Mediterráneo y Atlántico adyacente tal como, en la actualidad, lo hace $C$. neritea. 


\section{INTRODUCCIÓN}

Actualmente, aparte de la reconocida utilidad de la protoconcha para la sistemática de taxones de categoría supraespecífica (Bandel, 1993, 1996; Bandel y Kowalke, 1997), se acepta también que ciertas diferencias morfológicas entre protoconchas permiten discriminar especies en el caso de teleoconchas muy similares (Gili, 1992; Buochet y Warén, 1993; Solsona y Martinell, en prensa). Para una más amplia discusión véase Hoagland y Robertson (1988) y Bouchet (1989).

En la literatura paleontológica referente al Plioceno de las regiones mediterránea y atlántica adyacente, se considera que los representantes del género Cyclope Risso, 1826 pertenecen a la especie actual $C$. neritea (Linnaeus, 1758). En efecto, la similitud morfológica de las formas adultas fósiles y actuales es casi total, y sus ligeras diferencias son del mismo rango de las que se suelen hallar entre poblaciones de una misma especie.

La reproducción y desarrollo de Cyclope neritea se conoce bien ((Boulhic y Tardy, 1986b; Le Roux, 1994): las hembras depositan cápsulas ovígeras que generalmente contienen un único huevo, la larva se desarrolla y sufre la metamorfosis en su interior y eclosiona en forma de joven reptante (desarrollo intracapsular, en el sentido de Bouchet, 1989). La morfología de la protoconcha, que sólo se observa en los ejemplares inmaduros, refleja este tipo de desarrollo (Shuto, 1974; Jablonski y Lutz, 1980 y 1983) (Fig. 1): paucispiral (1,5 vueltas), obtusa, con núcleo grueso y prominente, y con una relación diámetro/número de vueltas (dp/vol) entre 0,43 y 0,48 .

Si las formas fósil y actual correspondieran a lo que se espera de una especie evolutiva (véase González Donoso, 1995), es decir, pertenecieran a una misma línea de descendencia sin discontinuidad en el tiempo, su desarrollo larvario debería ser el mismo. Así pues, la protoconcha de la forma fósil debería presentar una morfología con el mismo patrón ontogenético que el de la especie actual. En la bibliografía consultada no se ha hallado ningún dato sobre la protoconcha de la supuesta $C$. neritea del Plioceno, excepto en Ferrero y Merlino (1992) en donde se figura un juvenil con protoconcha, de la región de Asti (Piemonte, NO de Italia), sin ningún comentario adicional. Por nuestra parte, en sedimento del Plioceno Inferior procedente de Monteu Roero (Piemonte, NO de Italia), hemos hallado formas juveniles de Cyclope con la protoconcha conservada junto a formas adultas que presentan el ápice seccionado. Por esta coincidencia en el tiempo y en el espacio, hemos asumido que unas y otras se corresponden. La morfología de estas protoconchas es multispiral (2,5 vueltas), bastante aguda, con núcleo pequeño no prominente, y con relación $\mathrm{dp} / \mathrm{vol}$ de 0,22 . Se trata, por tanto, de una morfología totalmente distinta de la de las formas actuales (Fig. 1). La protoconcha del ejemplar juvenil figurado por Ferrero y Merlino (1992) es similar. Este conjunto de caracteres de la protoconcha denota un proceso ontogenético en la fase larvaria distinto del de la especie actual. Por lo tanto, aún en el caso de haber coincidido ambas formas de Cyclope en algún momento, hubiera existido una barrera reproductora entre ellas. Es decir, dichas formas no pertenecen a la misma línea de descendencia, puesto que el cambio en el tipo de desarrollo crea una discontinuidad. La forma pliocénica de Cyclope no es conespecífica con la actual, es decir, nos hallamos ante dos especies distintas. El nombre C. nerited debe conservarse sólo para la forma viviente, ya que Linnaeus lo utilizó en este sentido.

En la bibliografía consultada referente a la malacofauna del Neógeno, solo hemos hallado una cita de Cyclope no atribuida a C. neritea. Bevilacqua (1928) describe una nueva especie, Cyclonassa migliorinii (= Cyclope migliorinii), para el Plioceno y Pleistoceno de la isla de Rodas (Grecia), diferenciándola explícitamente de la especie actual. Aunque la autora no hace referencia a la protoconcha de su especie, y el holotipo tampoco presenta esta estructura (Pinna y Spezia, 1978), hemos considerado que las formas halladas por nosotros en Monteu Roero se corresponden con esta especie, ya que la coincidencia entre los adultos es total. De todas maneras, en nuestra opinión, C. migliorinii, si bien está correctamente definida según la normativa, su descripción original adolece de la falta de información con relación a la protoconcha.

En este trabajo se comentan inicialmente los caracteres distintivos del género Cyclope, se redescribe C. migliorinii (Bevilacqua, 1928) y se hace una comparación exhaustiva con la especie actual. Asímismo, se discuten aspectos paleoecológicos, paleobiogeográficos y estratigráficos de la especie pliocénica.

\section{PALEONTOLOGÍA SISTEMÁTICA}

\author{
CLASE GASTROPODA Cuvier, 1797 \\ SUBCLASE PROSOBRANCHIA Milne Edwards, 1848 \\ ORDEN NEOGASTROPODA Thiele, 1929 \\ Familia Nassariidae Iredale, 1916 \\ Género Cyclope Risso, 1826
}

Especie tipo: Buccinum neriteum Linnaeus, 1758.

\section{Diagnosis}

Concha pequeña, sólida, discoidal, con perfil lenticular, convexa dorsalmente y más plana ventralmente, lisa. Espira normalmente truncada en los adultos, observándose sólo las últimas 2 vueltas de espira, las cuales se superponen casi totalmente sobre las anteriores de manera que la última vuelta forma casi toda la concha. Abertura pequeña, sub-circular piriforme, con columela lisa muy cóncava y labro fuertemente curvado. Interior del labro con pequeños dentículos o liso y con un engrosamiento externo. La callosidad columelar cubre toda la región ventral. Canal sifonal muy corto (no sobresale de la última vuelta) y estrecho, sin pliegue columelar (Fig. 2).

\section{Observaciones}

Hay varios aspectos morfológicos característicos de este género: 


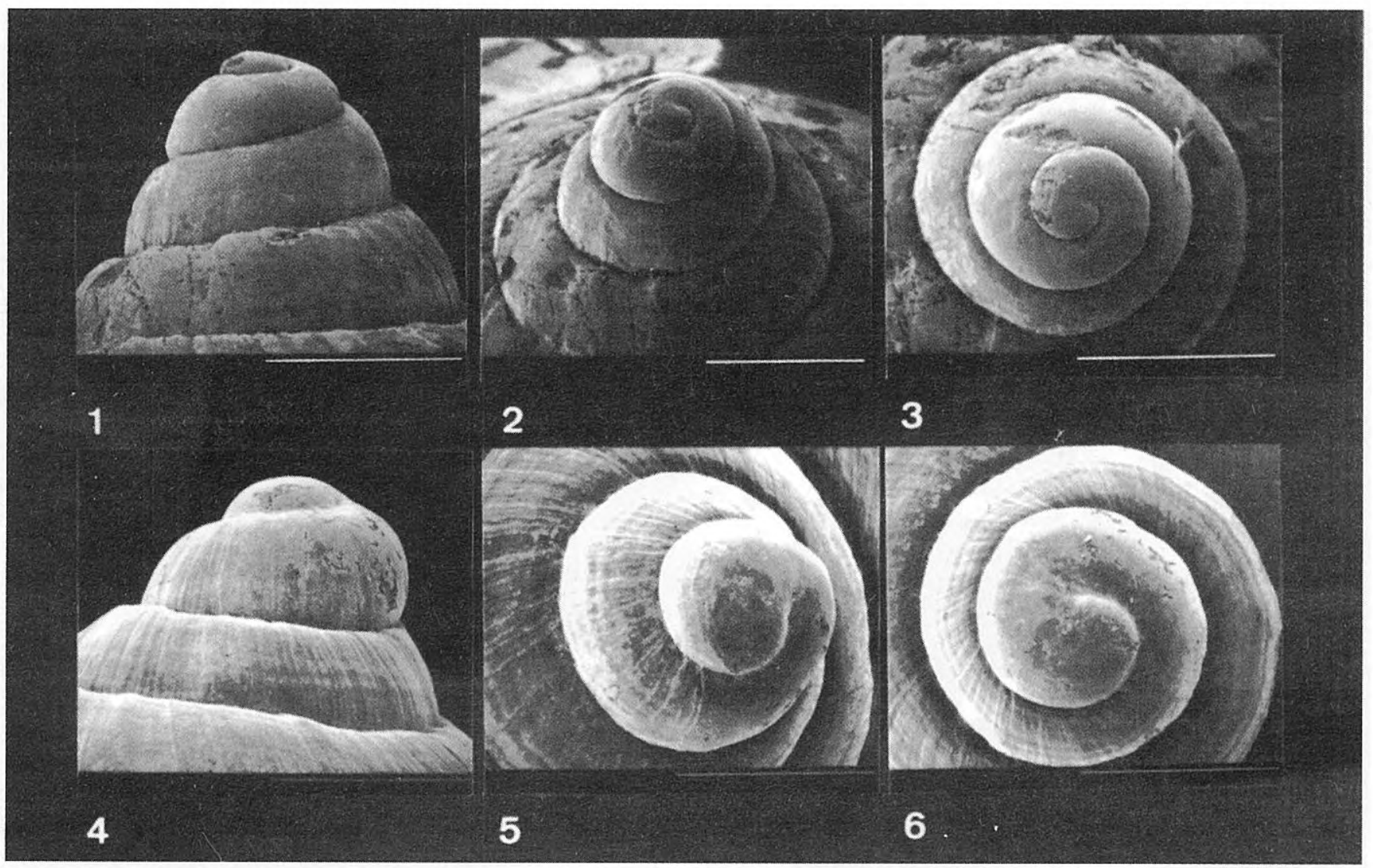

Figura 1. Protoconchas de Cyclope migliorinii (Bevilacqua, 1928) y de C. neritea (Linnaeus, 1758), figuradas según la norma propuesta por Gili y Martinell (1992). 1 - 3. C. migliorinii, Plioceno Inferior, Monteu Roero (Piemonte, NO Italia). Col. J. Martinell, Dep. d'Estratigrafia i Paleontologia, Universitat de Barcelona. 4 - 6. C. neritea, actual, Delta de l'Ebre (Tarragona, NE España). Col. J. Martinell, Dep. d'Estratigrafia i Paleontologia, Universitat de Barcelona. Escala gráfica: $500 \mu \mathrm{m}$.

I. La gran superposición de las vueltas de espira (Fig. 3) da a este género un aspecto similar al de algunos arqueogasterópodos (p. ej. Umbonium), pero ello se consigue por la eliminación del ápice (protoconcha y primeras vueltas de espira) y no por un enrollamiento homogéneo de toda la concha. Como se observa en las formas juveniles, las primeras vueltas de teleoconcha se enrollan de la misma forma que en Nassarius, es decir, cada vuelta cubre aproximadamente la mitad de la anterior, pero a partir de la tercera vuelta la superposición se hace mucho mayor. Esto permite reconocer estas formas jóvenes como pertenecientes al género Cyclope respecto a los juveniles del género Nassarius (Fig. 3).

II. La sección del ápice no es accidental sino un proceso activo del animal. En individuos que han llegado al máximo de desarrollo de la concha y todavía no han eliminado el ápice, es habitual observar una concavidad en la parte ventral de la espira que corresponde a la zona de disolución (Fig. 3). Una vez completada la eliminación, la cicatriz queda cubierta por una fina callosidad que es una expansión de la callosidad columelar. Este mismo proceso se observa en Nassarius (Plicarcularia) circumcinctus (A. Adams, 1852) (Oliverio y Tringali, 1992).

III. La abertura de la concha se sitúa en un plano casi perpendicular al eje de enrollamiento de la concha, mientras que lo normal, incluso en las formas tipo Umbonium, es que el plano de apertura sea más o menos paralelo a este eje. Esto se consigue por una desviación de la sutura hacia la periferia en el último cuarto de vuelta. (Fig. 3). El resultado es una geometría de la concha y, como consecuencia, una posición de vida del animal, excepcional entre los neogasterópodos (Vermeij, 1971).

IV. El engrosamiento externo del labro es una estructura distinta de la habitual variz labial en nasáridos y otros gasterópodos. La variz labial consiste en un engrosamiento más o menos definido de la periferia del labro construido a partir de la capa externa de la concha, en discontinuidad con la capa interna. En Cyclope, así como en el subgénero Nassarius (Plicarcularia) Thiele, el engrosamiento consiste en una expansión de la capa interna de la concha sobre el borde externo del labro. El material que lo forma es el mismo que el de la callosidad columelar. Al ser una estructura superpuesta, queda claramente delimitada respecto a la parte dorsal de la concha por un fino surco. Para esta estructura especial, no homóloga de la variz labial, proponemos el nombre de "callosidad labral". Normalmente, cuando existe callosidad labral, ésta se halla fusionada con la callosidad columelar por la zona adapical de la abertura y por encima del canal sifonal. 


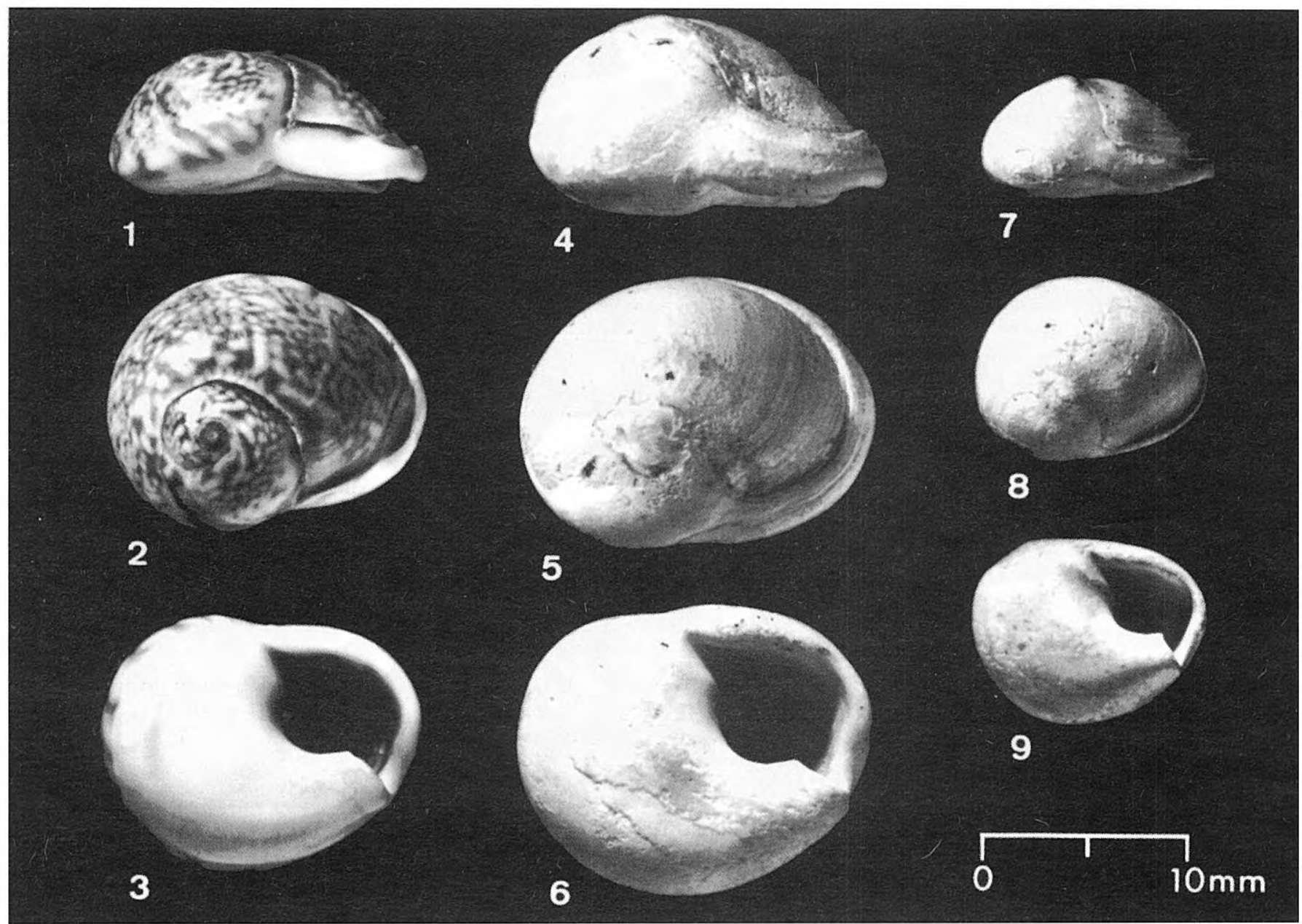

Figura 2. Teleoconchas de Cyclope neritea (Linnaeus, 1758) y Cyclope migliorinii (Bevilacqua, 1928). 1 - 3. C. neritea, actual, Camargue (SE Francia). Col. C. Gili. 4 - 6. C. migliorinii, Plioceno Inferior, Velerín (Málaga, S España). Col. J. Martinell, Dep. d'Estratigrafia i Paleontologia, Universitat de Barcelona. 7 - 9. C. migliorinii, Plioceno Inferior, Monteu Roero (Piemonte, NO Italia). Col. J. Martinell, Dep. d'Estratigrafia i Paleontologia, Universitat de Barcelona.

\section{Cyclope migliorinii (Bevilacqua, 1928) \\ Fig. 1, Fig. 2}

1901 Cyclonassa neritea (Linné); Cossmann, 217, lám. 1. 9, fig. 11.

1904 Cyclonassa neritea (L.); Sacco, 72, lám. 17, figs. 6 - 7.

1911 Cyclonassa neritea L; Cerulli-Irelli, 254, lám. 23, figs. $57-58$.

1928 Cyclonassa migliorinii sp. n. Bevilacqua, 173, lám. 3, figs. $11 \mathrm{a}-\mathrm{e}$.

1959 Nassa (Cyclonassa) neritea Linné; Zbyszewski, 96.

1974 Cyclope (Cyclope) neritea (L. 1766); Malatesta, 324, lám. 26, figs. $3 \mathrm{a}$ - b.

1975 Cyclonassa neritea (Linné); Fekih, 125, lám. 39, figs. $2 \mathrm{a}-\mathrm{d}$.

1975 Cyclope (C.) neritea (Linné); Pavia, 113.

1978 Cyclonassa migliorinii Bevilacqua, 1928; Pinna y Spezia, 138, lám. 45, figs. 1 - 1 b.

1979 Cyclope (C.) neritea (Linné); Montefameglio et al., 190.

1992 Cyclope (Cyclope) neritea (L., 1758); Cavallo y Reppeto, 104, fig. 242.
1992 Cyclope (C.) neritea (Linneo); Ferrero y Merlino, 129, lám. 1 , fig. 9.

1995b Cyclope neritea (Linné, 1758); Vera-Peláez et al., 110.

(En esta sinonimia sólo se han hecho constar aquellos trabajos de los cuales se ha podido consultar el material original, aunque no contengan figuras, y aquellos que presentan figuras claras. Se han omitido las citas que no presentan el material figurado o las figuras no son suficientemente claras).

Holotipo: Bevilacqua 1928, pág. 173, Lám. 3, figs. 11 a, b, c y d. Museo Civico di Storia Naturale di Milano, n ${ }^{\circ} 2319$.

\section{Material examinado}

Monteu Roero (Piemonte, NO Italia), Plioceno Inferior (Zancliense), 46 ejemplares; Velerín (Malaga, S España), Plioceno Inferior (Zancliense), 77 ejem.; Pedassos (Peloponeso, Grecia), Plioceno medio (Plasenciense), 19 ejem.; San Giacomo (Piemonte, NO Italia), Plioceno Inferior (Zancliense), 2 ejem.; San Pedro de Muel (Mina, O 

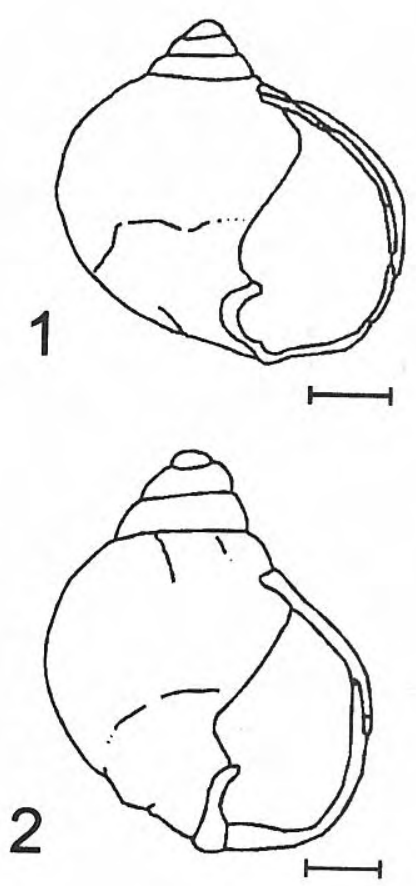
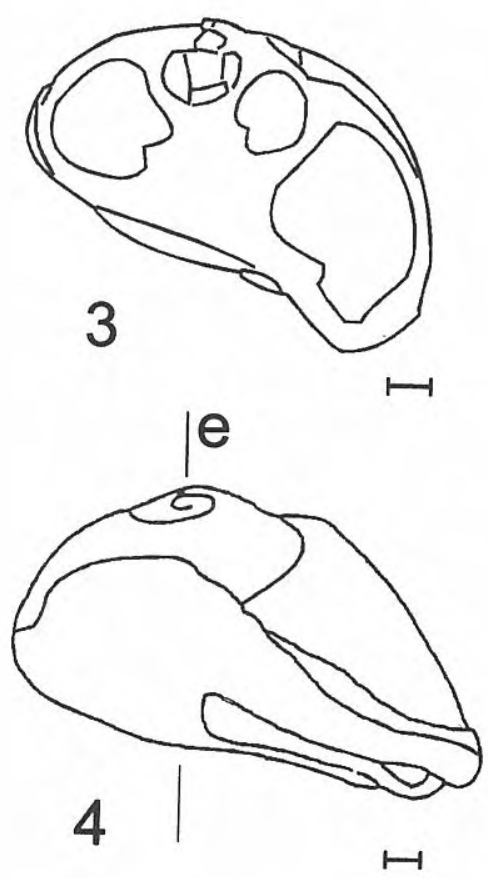
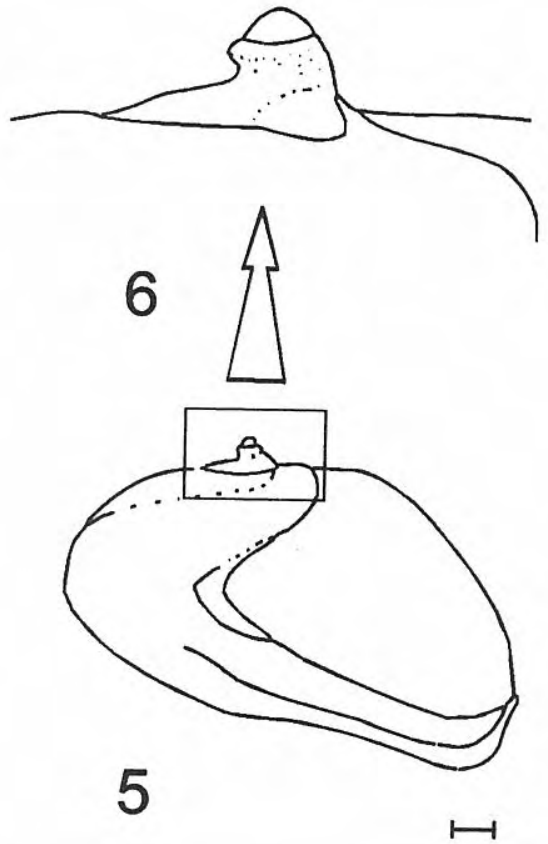

Figura 3. Dibujos esquemáticos. 1. Cyclope migliorinii (Bevilacqua, 1928), ejemplar juvenil, Plioceno Inferior, Monteu Roero (Piemonte, NO Italia). 2. Nassarius aff. gibbosulus (Linnaeus, 1758), ejemplar juvenil, Plioceno Inferior, El Papiol (Barcelona, NE España). 3. Cyclope neritea (Linnaeus, 1758), sección de un ejemplar adulto, actual, Delta de l'Ebre (Tarragona, NE España). 4. C. neritea, ejemplar adulto, actual, Camargue (SE Francia). 5. C. neritea, ejemplar adulto con el ápice en proceso de seccionamiento, actual, Delta de l'Ebre (Tarragona, EN España). 6. C. neritea, detalle del ápice del ejemplar anterior. Escala gráfica: $1 \mathrm{~mm}$. e: eje de enrollamiento de la espira.

Portugal), Plioceno medio (Plasenciense), 1 ejem.

\section{Diagnosis}

La misma que para el género, con la sutura de la última vuelta en los adultos muy próxima a la de la penúltima vuelta, y con protoconcha de 2,5 vueltas.

\section{Descripción}

Protoconcha: consta de 2,5 vueltas lisas y convexas, con núcleo (primera $1 / 2$ vuelta) pequeño $(0,1 \mathrm{~mm}$ de diámetro en el ejemplar figurado), no prominente, con crecimiento espiral regular. El tránsito a la teleoconcha sólo se distingue por la aparición de las estrías de crecimiento y por un cambio en la textura de la concha (Fig. 1).

Teleoconcha: Discoidal, perfil lenticular, sin ornamentación y con estrías de crecimiento opistocírticas. La última vuelta cubre la mayor parte de la concha, su línea de sutura se halla casi en contacto con la de la penúltima vuelta. Concha muy convexa dorsalmente, con una ligera gibosidad, mucho menos convexa ventralmente. Espira truncada en los adultos. Abertura sub-circular piriforme, corta y ancha, con estrecho y profundo canal anal. Canal sifonal muy corto, abierto, que no sobresale de la última vuelta. Columela lisa, muy cóncava, sin cresta columelar en el borde del canal sifonal. Labro engrosado externamente por una gruesa callosidad labral, internamente puede presentar cortos dentículos (2 a 13). La callosidad columelar se extiende sobre toda la región ventral y puede alcanzar, también ventralmente, la zona de sección de la espira a partir de la parte adapical de la abertura.
Para el conjunto de los especímenes estudiados, los valores medios de los parámetros medidos son: altura $(\mathrm{H}) 7$, $34 \mathrm{~mm}$, diámetro (D) $13,6 \mathrm{~mm}$, anchura (A) $10,85 \mathrm{~mm}$ y altura de la abertura (Ho) $8,71 \mathrm{~mm}$ (Tab. 1).

Variabilidad de la teleoconcha: la morfología del material de las distintas muestras estudiadas es muy similar, sólo pocos caracteres presentan alguna variación. Los dentículos labrales normalmente se hallan ausentes en Piemonte y Pedassos (solo 3 y 4 ejemplares los presentan, respectivamen-te), mientras que en Málaga los tienen casi la mitad de los ejemplares. La anchura de la callosidad labral también varía, pero en la mayoria (72 de 84 ejemplares medidos) es muy ancha. Son muy pocos los individuos en los que la callosidad columelar no se expande hacia el ápice truncado.

Las dimensiones, por el contrario, son bastante variables según el origen del material, aunque en cada localidad las medidas son más homogéneas. Los ejemplares de Velerín son los mayores (diámetro medio de $16,18 \mathrm{~mm}$ ). Los de Pedassos presentan los valores menores (diámetro medio de $8,47 \mathrm{~mm}$ ). En Monteu Roero y San Giacomo se dan valores intermedios (diámetro medio de $11,59 \mathrm{~mm}$ ), aunque se ha hallado un ejemplar muy grande (diámetro de $18,05 \mathrm{~mm}$ ). A pesar de la variación en el tamaño, las relaciones entre los distintos parámetros son bastante homogéneas en las distintas localidades (Tab. 2). La comparación de los valores medios de la relación $\mathrm{D} / \mathrm{H}$, entre todas las parejas de localidades, mediante el test $\mathrm{T}$ - Student (a un nivel de significación $=0,05)$, indica que las diferencias no son estadísticamente significativas. 


\section{Cyclope migliorinii (Bevilacqua, 1928)}

\begin{tabular}{lccccccc} 
& H & D & A & Ho & D/H & A/D & Ho/H \\
\hline \hline Número de datos & 91 & 91 & 91 & 91 & 91 & 91 & 91 \\
Valor máximo & 10,10 & 18,80 & 14,60 & 12,15 & 2,07 & 0,88 & 1,46 \\
Valor mínimo & 3,70 & 7,65 & 5,60 & 4,20 & 1,63 & 0,65 & 0,93 \\
Media & 7,34 & 13,60 & 10,85 & 8,71 & 1,86 & 0,80 & 1,19 \\
Mediana & 7,90 & 14,75 & 11,85 & 9,35 & 1,86 & 0,79 & 1,18 \\
Desviación típica & 1,88 & 3,38 & 2,82 & 2,32 & 0,08 & 0,03 & 0,09 \\
Varianza & 3,53 & 11,44 & 7,98 & 5,37 & 0,01 & 0,00 & 0,01 \\
\hline
\end{tabular}

\section{Cyclope neritea (Linnaeus, 1758)}

\begin{tabular}{lccccccc} 
& H & D & A & Ho & D/H & A/D & Ho/H \\
\hline \hline Número de datos & 68 & 68 & 68 & 68 & 68 & 68 & 68 \\
Valor máximo & 7,65 & 15,90 & 12,45 & 9,80 & 2,17 & 0,82 & 1,36 \\
Valor mínimo & 4,35 & 8,70 & 6,90 & 5,05 & 1,69 & 0,77 & 1,01 \\
Media & 5,96 & 11,72 & 9,20 & 6,88 & 1,97 & 0,79 & 1,15 \\
Mediana & 5,75 & 11,23 & 8,83 & 6,50 & 1,97 & 0,78 & 1,15 \\
Desviación típica & 0,99 & 1,93 & 1,51 & 1,26 & 0,09 & 0,01 & 0,08 \\
Varianza & 0,98 & 3,73 & 2,28 & 1,58 & 0,01 & 0,00 & 0,01 \\
\hline
\end{tabular}
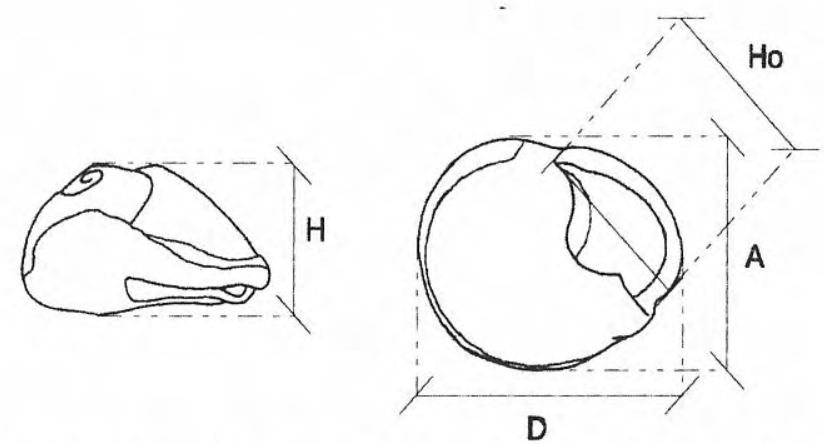

Tabla 1. Resumen global de los valores (en mm) de los parámetros medidos en las dos especies que se comparan. $\mathrm{H}$ = altura; $\mathrm{D}=$ diámetro; $\mathrm{A}$ = anchura; Ho = altura de la abertura. En el gráfico se esquematiza el significado de cada parámetro, según han sido medidos.

Comparación con Cyclope neritea (Linnaeus, 1758) (Fig. 1, Fig. 2)

Los datos referentes a la especie actual $C$. neritea se han obtenido a partir del estudio del material siguiente: Tavira (Algarve, S Portugal), 6 ejem.; Mar Menor (Murcia, E España), 2 ejem.; Delta de l'Ebre (Tarragona, NE España), 53 ejem. (3 juv.); Étang de Leucate (Aude, SE Francia), 38 ejem. (8 juv.); Camargue (Bouches-du-Rhône, NE Francia), 18 ejem.; Costinesti (Rumanía), 4 ejem.

Tal como se ha mencionado anteriormente, la diferencia básica entre estas dos especies es la morfología de su protoconcha. La de $C$. neritea tiene sólo 1,5 vueltas lisas, núcleo grueso y prominente $(0,3 \mathrm{~mm}$ de diámetro en el individuo figurado), espira de crecimiento algo irregular, y un perfil muy convexo. El tránsito a la teleoconcha está marcado por la aparición de 6 cordoncillos muy débiles que se pierden muy pronto.

\section{Cyclope migliorinii (Bevilacqua, 1928)}

\begin{tabular}{|c|c|c|c|c|c|c|c|}
\hline & $\mathrm{H}$ & D & A & Ho & $\mathrm{D} / \mathrm{H}$ & $A / D$ & $\mathrm{Ho} / \mathrm{H}$ \\
\hline Número de datos & 50 & 50 & 50 & 50 & 50 & 50 & 50 \\
\hline Valor máximo & 10,10 & 18,80 & 14,60 & 12,15 & 2,01 & 0,85 & 1,33 \\
\hline Valor mínimo & 6,95 & 13,30 & 10,75 & 8,55 & 1,63 & 0,77 & 0,98 \\
\hline Media & 8,76 & 16,18 & 13,02 & 10,47 & 1,85 & 0,80 & 1,20 \\
\hline Mediana & 8,88 & 16,43 & 13,23 & 10,65 & 1,86 & 0,81 & 1,19 \\
\hline Desviación típica & 0,67 & 1,18 & 0,91 & 0,84 & 0,08 & 0,02 & 0,07 \\
\hline Varianza & 0,45 & 1,39 & 0,83 & 0,70 & 0,01 & 0,00 & 0,00 \\
\hline
\end{tabular}

MONTEU ROERO + SAN GIACOMO (Piemonte, NO Italia)

\begin{tabular}{lccccccc} 
& H & D & A & Ho & D/H & A/D & Ho/H \\
\hline \hline Número de datos & 24 & 24 & 24 & 24 & 24 & 24 & 24 \\
Valor máximo & 10,00 & 18,05 & 14,25 & 10,45 & 2,07 & 0,88 & 1,46 \\
Valor mínimo & 3,70 & 7,65 & 6,00 & 5,15 & 1,67 & 0,76 & 1,04 \\
Media & 6,20 & 11,59 & 9,25 & 7,44 & 1,88 & 0,80 & 1,21 \\
Mediana & 6,23 & 11,33 & 9,10 & 7,60 & 1,88 & 0,79 & 1,21 \\
Desviación típica & 1,27 & 2,09 & 1,67 & 1,20 & 0,10 & 0,03 & 0,10 \\
Varianza & 1,60 & 4,37 & 2,80 & 1,45 & 0,01 & 0,00 & 0,01 \\
\hline
\end{tabular}

PEDASSOS (Peloponeso, S Grecia)

\begin{tabular}{lccccccc} 
& H & D & A & Ho & D/H & A/D & Ho/H \\
\hline \hline Número de datos & 16 & 16 & 16 & 16 & 16 & 16 & 16 \\
Valor máximo & 5,20 & 9,55 & 7,30 & 6,00 & 2,00 & 0,79 & 1,23 \\
Valor mínimo & 4,00 & 7,65 & 5,60 & 4,20 & 1,71 & 0,65 & 0,93 \\
Media & 4,56 & 8,47 & 6,44 & 5,05 & 1,86 & 0,76 & 1,11 \\
Mediana & 4,48 & 8,33 & 6,35 & 4,90 & 1,86 & 0,77 & 1,13 \\
Desviación típica & 0,35 & 0,67 & 0,52 & 0,57 & 0,08 & 0,03 & 0,08 \\
Varianza & 0,12 & 0,45 & 0,27 & 0,33 & 0,01 & 0,00 & 0,01 \\
\hline
\end{tabular}

Cyclope neritea (Linnaeus, 1758)

DELTA DE L'EBRE (Tarragona, NE España)

\begin{tabular}{lccccccc} 
& H & D & A & Ho & D/H & A/D & Ho/H \\
\hline \hline Número de datos & 50 & 50 & 50 & 50 & 50 & 50 & 50 \\
Valor máximo & 7,15 & 12,60 & 10,00 & 7,90 & 2,17 & 0,82 & 1,36 \\
Valor mínimo & 4,35 & 8,70 & 6,90 & 5,05 & 1,69 & 0,77 & 1,01 \\
Media & 5,47 & 10,69 & 8,39 & 6,23 & 1,96 & 0,79 & 1,14 \\
Mediana & 5,48 & 10,55 & 8,33 & 6,30 & 1,96 & 0,79 & 1,13 \\
Desviación típica & 0,60 & 0,96 & 0,73 & 0,68 & 0,10 & 0,01 & 0,08 \\
Varianza & 0,36 & 0,92 & 0,53 & 0,46 & 0,01 & 0,00 & 0,01 \\
\hline
\end{tabular}

CAMARGUE (SE Francia)

\begin{tabular}{lccccccc} 
& H & D & A & Ho & D/H & A/D & Ho/H \\
\hline \hline Número de datos & 18 & 18 & 18 & 18 & 18 & 18 & 18 \\
Valor máximo & 7,65 & 15,90 & 12,45 & 9,80 & 2,15 & 0,81 & 1,29 \\
Valor mínimo & 6,85 & 13,70 & 10,90 & 7,80 & 1,86 & 0,77 & 1,09 \\
Media & 7,35 & 14,57 & 11,45 & 8,68 & 1,98 & 0,79 & 1,18 \\
Mediana & 7,38 & 14,50 & 11,43 & 8,65 & 1,99 & 0,78 & 1,17 \\
Desviación típica & 0,24 & 0,57 & 0,41 & 0,45 & 0,08 & 0,01 & 0,05 \\
Varianza & 0,06 & 0,33 & 0,17 & 0,20 & 0,01 & 0,00 & 0,00 \\
\hline
\end{tabular}

Tabla 2. Resumen de los valores (en $\mathrm{mm}$ ) de los parámetros medidos en las dos especies, agrupados por yacimientos. $\mathrm{H}=$ altura; $\mathrm{D}=$ diámetro; $\mathrm{A}=$ anchura; Ho = altura de la abertura. (Véase significado de estos parámetros en Tabla 1).

$\mathrm{Al}$ igual que $C$. migliorinii, $C$. neritea elimina el ápice de la concha al llegar al final de su crecimiento, sólo se han encontrado un pequeño número de indivíduos que todavía lo conservan. Esto plantea la cuestión de cuales 


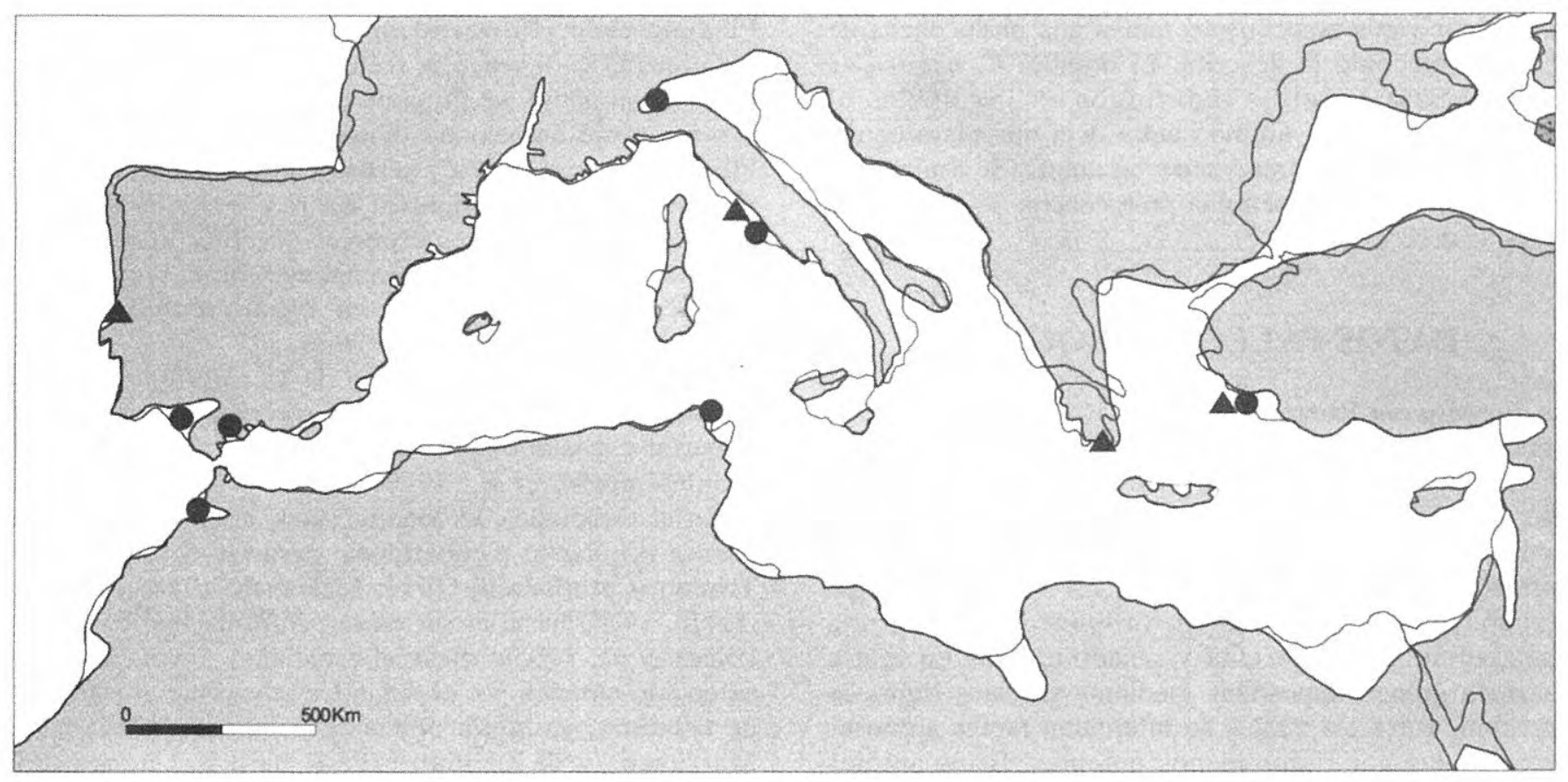

Figura 4. Mapa de las cuencas pliocénicas (según Solsona, 1998) con la distribución de Cyclope migliorinii (Bevilacqua, 1928). Plioceno inferior (Zancliense) $\Delta$ Plioceno medio (Plasenciense)

son los caracteres en que debe basarse la diferenciación entre las formas adultas de las dos especies.

Muchos autores (véase la sinonimia) indican que las formas fósiles (que consideran como $C$. neritea) se caracterizan por tener dimensiones mayores que las vivientes. Cerulli-Irelli (1911) da 19,5 mm como valor máximo para su material del Plioceno, mientras que el valor máximo hallado en la bibliografía para las formas actuales es de 17 mm (Parenzan, 1970; Le Roux, 1994). El mayor valor del diámetro (D) medido por nosotros para $C$. neritea es de $16,3 \mathrm{~mm}$ (Leucate, Francia) y no se han hallado diferencias estadísticamente significativas de las medias de la relación $\mathrm{D} / \mathrm{H}$ entre las dos poblaciones de $C$. neritea medidas (mediante el test $\mathrm{t}$ - Student, a un nivel de significación $=0,05)$. Como puede verse (Tab. 2), las dimensiones de ambas especies varían según la localidad de origen. Así, mientras que $C$. migliorinii alcanza medidas máximas mayores que $C$. neritea, esto no es constante en general. Los ejemplares de $C$. neritea de algunas localidades son mayores que los de $C$. migliorinii de otras localidades. Por tanto, este carácter no sirve para la diferenciación. A pesar de ello, se observan diferencias poblacionales en algunos parámetros. Así, la comparación mediante el test t Student (a un nivel de significación $=0,05$ ) de los valores medios de las relaciones $\mathrm{D} / \mathrm{H}$ y A/D de las dos especies (Tab. 1), indica que las diferencias son estadísticamente significativas. Es decir, para un mismo valor de D el valor de $\mathrm{H}$ en $C$. neritea es menor que en $C$. migliorinii, y el valor de A es un poco mayor en $C$. migliorinii que en C. neritea.

Por otra parte, Bevilacqua (1928, p. 173) indica las siguientes diferencias morfológicas entre $C$. migliorinii y $C$. neritea: un mayor cubrimiento de la espira por parte de la última vuelta, la región ventral más convexa y la presencia de dentículos labrales, en la especie fósil. Otros detalles que se pueden añadir, a partir del material que hemos estudiado, son: última vuelta más uniformemente convexa en los fósiles y más plana en los actuales, callosidad labral más ancha y callosidad columelar más marcadamente dilatada sobre la espira en la forma pliocena.

La mayoría de estas diferencias no representan una discontinuidad morfológica entre $C$. migliorinii y $C$. neritea. Los dentículos labrales, el grosor de la callosidad labral y la expansión de la callosidad columelar no son exclusivas de ninguna de las dos especies. Los dentículos del labro también se han observado en 7 ejemplares de $C$. neritea de Camargue (Francia), la expansión de la callosidad columelar sobre la espira se ha observado en 23 de los 68 especímenes de $C$. neritea, la callosidad labral de $C$. neritea puede ser tan ancha como en $C$. migliorinii (en 39 de los 68 individuos medidos). Con relación a los otros detalles mencionados, la variabilidad de $C$. neritea es también grande, tal como describe Pallary (1919), y se da una amplia coincidencia con $C$. migliorinii.

No obstante, uno de estos caracteres, aparte de la protoconcha, parece ser diferente, de forma constante, en las dos especies: en la forma actual, la última vuelta cubre una parte menor de la penúltima vuelta que en la forma fósil. En $C$. neritea la sutura de la última vuelta queda claramente separada de la sutura de la penúltima vuelta, mientras que en $C$. migliorinii ambas suturas se hallan casi en contacto y, en algun caso, la última vuelta llega a cubrir la sutura de la anterior (compárense las figuras de cada especie en la Fig. 2).

En consecuencia, para las formas adultas, se propone considerar como C. migliorinii aquellos individuos en los cuales la última vuelta cubre totalmente la anterior, y se 
asume que estos especímenes tenían una protoconcha de 2,5 vueltas como la descrita. El nombre $C$. neritea se reserva para aquellos individuos en los cuales el recubrimiento de la última vuelta deja una clara banda adapical de la penúltima vuelta sin cubrir. Se asume que estos especímenes tienen una protoconcha de 1,5 vueltas como la descrita.

\section{DATOS PALEOECOLÓGICOS}

En Monteu Roero (Piemonte, Italia), Pavia (1975) concluye que la malacofauna fósil es el resultado de la mezcla de varias biocenosis. Este autor sitúa Cyclope en dos de ellas, correspondientes a arenas costeras bien calibradas y arcillas arenosas lagunares protegidas, con influencia de aguas continentales. En Velerín (Málaga, España), Vera Peláez et al. (1995a) describen una malacofauna transportada y resedimentada en facies detrítica gruesa, depositada mediante potentes flujos de tracción, entre los cuales se intercalan facies arenosas depositadas por flujos menos potentes. Estos autores asocian las facies detríticas con la influencia de corrientes fluviales en zona costera. Koutsouveli (1987 y observaciones personales), indica que el yácimiento de Pedassos (Peloponeso, Grecia) corresponde a una acumulación de fauna marina con una importante representación de fauna de aguas salobres. Estos datos permiten suponer que C. migliorinii ocupaba zonas marinas con influencia más o menos directa, pero siempre importante, de aguas dulces.

Todas estas asociaciones faunísticas corresponden a ambientes de aguas someras. Pavia (1975), para Monteu Roero, les asigna una profundidad entre 6 y $20 \mathrm{~m}$. Este hábitat ocupado por $C$. migliorinii no parece muy distinto del ambiente típico donde vive $C$. neritea en la actualidad. Boulhic y Tardy (1986a) concluyen que $C$. neritea es una forma típica de lagunas y marismas costeras, donde se halla en altas densidades de población. Es una especie adaptada a amplias variaciones de salinidad y temperatura. La tanatocenosis reciente de Tavira (Portugal) presenta una estructura y una asociación faunística equivalente a la de los depósitos del Plioceno de Pedassos (Grecia) (observaciones personales).

Parece que la especie fósil C. migliorinii tenía los mismos requerimientos ecológicos que la actual $C$. neritea.

\section{REPARTICIÓN ESTRATIGRÁFICA Y GEOGRÁFICA}

C. migliorinii es, cronológicamente, la primera especie aparecida de su género. Se localiza a partir del Plioceno Inferior pero el momento de su extinción no está bien delimitado. Bevilacqua (1928) indica la presencia de su especie desde el Plioceno (sensu lato) hasta el Calabriense (Pleistoceno Inferior). El material que hemos estudiado pertenece al Zancliense (Plioceno Inferior) y al
Plasenciense (Plioceno medio). Parece claro que actualmente $C$. neritea ha reemplazado a $C$. migliorinii. Provisionalmente, se propone considerar $C$. migliorinii como especie que existió durante todo el Plioceno y el Pleistoceno Inferior, y $C$. neritea como una especie muy reciente. Por el momento, no hay evidencia de la coexistencia de las dos especies en el tiempo y en el espacio. Se puede asumir, también de forma provisional, que $C$. neritea apareció en algún momento del Pleistoceno posterior al Calabriense.

La distribución geográfica de C. migliorinii en el Plioceno (Fig. 4) incluye el Mar Egeo (Bevilacqua, 1928; material estudiado) y el norte de Italia (Pavia, 1975; Montefameglio et al., 1979; Ferrero y Merlino, 1992; material estudiado), el Mediterráneo oriental, y toda la cuenca occidental mediterránea, desde la costa del Mar Tirreno (Cerulli-Irelli, 1911; Malatesta, 1974) y Túnez (Fekih, 1975) hasta el sur de la península Ibérica (Vera Peláez et al., 1995b; material estudiado). Podría haberse extendido también por el Atlántico adyacente al estrecho de Gibraltar, ya que las citas de Lecointre (1952) para Marruecos y de Landau (1984) para la Cuenca del Guadalquivir (SO España), corresponden probablemente a esta especie. Su presencia en la costa atlántica es segura en Portugal (Zbyszewski, 1959; material estudiado).

\section{DISCUSIÓN Y CONCLUSIONES}

El género Cyclope presenta algunas características notables y distintas de los otros nasáridos. Su forma discoidal de perfil lenticular, como consecuencia del enrollamiento de unas vueltas superpuestas en gran medida sobre las anteriores y del seccionamiento del ápice, y la posición de la abertura, casi perpendicular al eje de enrollamiento, es una arquitectura única entre los neogasterópodos.

Las formas del género Cyclope del Plioceno y Pleistoceno Inferior mediterráneos pertenecen a una especie distinta de la viviente en la actualidad en la misma región, $C$. neritea, con la cual fue confundida mayoritariamente. La especie fósil debe designarse como Cyclope migliorinii (Bevilacqua, 1928). La diferencia fundamental entre ambas es la morfología de su protoconcha, multispiral en la forma fósil y paucispiral en la forma actual. Aunque a nivel de población se observan diferencias cuantitativas en los parámetros de la teleoconcha, sus teleoconchas son cualitativamente casi idénticas. La diferencia más constante que se ha hallado es el mayor recubrimiento de la última vuelta sobre la penúltima en $C$. migliorinii. Los datos paleoecológicos de los yacimientos donde se encuentra $C$. migliorinii llevan a la conclusión de que, desde el punto de vista autoecológico, esta especie era equivalente a $C$. neritea. Ambas pueden considerarse especies gemelas (sibling species) por el hecho de que comparten los caracteres de la teleoconcha y los requerimientos ecológicos del animal adulto, pero se diferencian claramente por los de la protoconcha, lo cual indica diferentes modos de desarrollo embrionario-larvario. 
Con relación a este tipo de especies gemelas, en las cuales los adultos no conservan la protoconcha, se debe hacer una reflexión sobre el papel de los ejemplares tipo. Si los tipos deben servir para estabilizar la nomenclatura y el individuo elegido como holotipo es adulto, este holotipo podría no ser adecuado al propósito para el que fue designado. Aunque en ningún artículo del Codigo de Nomenclatura Zoológica se indique que el tipo deba ser un ejemplar adulto (la recomendación $73 \mathrm{C}$ señala la conveniencia de indicar el estado de desarrollo del holotipo), no parece conveniente que el holotipo de un gasterópodo sea simplemente una protoconcha o una forma juvenil poco desarrollada. Este estadio del desarrollo no presupone una teleoconcha adulta determinada. Son frecuentes las especies de nasáridos que no se distinguen en su estado larvario (protoconcha) o más juvenil sino en un estadio de desarrollo más avanzado. (De Renzi y Martinell, 1979; Martinell, 1982; Gili y Martinell, 1990; Gili, 1991). Por el contrario, en especies gemelas en las que los adultos conservan sus protoconchas, éstas son muy distintas y, en cambio, sus teleoconchas casi no se distinguen (Gili, 1992) con lo que elegir un ejemplar juvenil como holotipo tampoco sería correcto. Debería considerarse la necesidad, para los casos como el de C. migliorinii y $C$. neritea tratado en el presente trabajo, de que la descripción original de la especie contemplara los distintos estados de desarrollo, especialmente el estado larvario (protoconcha), siempre que hubiera suficiente seguridad de que todos ellos pertenecen a la misma serie ontogenética. En estos casos la descripción basada en un ejemplar, y solamente uno, el holotipo, tiene los efectos contrarios a aquellos para los cuales los tipos fueron propuestos.

C. migliorinii es una especie extinguida que vivió durante todo el Plioceno y parte del Pleistoceno en la región mediterránea y del Atlántico adyacente, aunque no hay suficientes datos para asegurar el momento de su extinción. En algún momento del Pleistoceno dio lugar a la actual $C$. neritea que la sustituyó en toda su área de distribución mediterránea. Esta repartición estratigráfica da una longevidad para la especie C. neritea muy inferior a la que se le suponía. Con ello se demuestra que la revisión sistemática del material tratado es un importante requisito previo en los estudios de tendencias macroevolutivas (Gili y Martinell, 1994).

El proceso de especiación implicado en la aparición de $C$. neritea a partir de $C$. migliorinii sigue la misma pauta ya observada en otros Nassariidae (Gili y Martinell, 1990; Gili, 1991 y 1992). A partir de especies con protoconcha multiespiral aparecen especies con protoconcha paucispiral que, al menos inicialmente, poseen teleoconchas idénticas.

\section{AGRADECIMIENTOS}

Nuestro agradecimiento al Dr. G. Pavia (Universidad de Torino) que nos proporcionó parte del material utilizado, y a las Dras. Rosa Domènech y Marta Solsona (Universitat de Barcelona) por sus comentarios críticos y ayuda en la elaboración del original. Hemos recibido consejo y ayuda del Servei de Microscòpia Electrònica (Universitat de Barcelona) y del Servei de Dibuix i Disseny Gràfic (Universitat de Barcelona, Divisió III) en la obtención de las imágenes del S.E.M. y de las figuras que acompañan el texto. Las macrofotografias de los ejemplares son obra del Sr. J. M. Moraleja. Este trabajo se enmarca en el Proyecto de Investigación DGICYT PB94-0946 y en la Acción Integrada Hipano-Portuguesa HP96-60.

\section{BIBLIOGRAFÍA}

Bandel, K. 1993. Caenogastropoda during Mesozoic times. Scripta Geologica, Special Issue 2, 7-56.

Bandel, K. 1996. Some heterostrophic gastropods from Triasic St. Cassian Formation with a discussion on the classification of the Allogastropoda. Paläontologische Zeitschrift, 7 (3/4), 325-365.

Bandel, K. \& Kowalke, T. 1997. Systematic value of the larval shell of fossil and modern Vanikoridae, Pickworthiidae and the genus Fossarus (Caenogastropoda, Mollusca). Berlines Geowissenschaftliche Abhandlungen, E 25, 3-29.

Bevilacqua, A. 1928. Studi sulla fauna fossile marina pliocenica e quaternaria dell'isola de Rodi (Egeo). Atti Società Italiana Scienze Naturali. Museo Milano, 67, 150-178.

Bouchet, P. 1989. A review of poecilogony in gastropods. Journal of Molluscan Studies, 55, 67-78.

Bouchet, P. \& Warén, A. 1993. Revision of the northeast Atlantic bathyal and abyssal Mesogastropoda. Bolletino Malacologico, suppl. 3, 580-840.

Bouhlic, M. et Tardy, J. 1986a. Cyclope neritea (Linné, 1758): Gastéropode Prosobranche nouvellement implanté sur les côtes de Charente-Maritime, éthologie, structure de la population. Haliotis, 15, 41-49.

Bouhlic, M. et Tardy, J. 1986b. Ponte, développement larvaire et éthologie des juveniles de Cyclope neritea (Linné, 1758). Haliotis, 15, 51-58.

Cavallo, O. e Repetto, G. 1992. Conchiglie Fossili del Roero. Atlante Iconografico. Associazione Naturalistica Piemontese, Memorie 2, Associazione Amici del Museo "Federico Eusebio", 1-251.

Cerulli-Irelli, S. 1911. Fauna malacologica Mariana. Parte quinta. Paleontographia Italica, 17, 243- 305. Museo Geologico della R. Università de Pisa, Forni Editore. Bologna.

Cossmann, M. 1901. Essais de paleoconchologie comparée, 4, 1-293. Paris.

De Renzi, M. y Martinell, J. 1979. Algunos aspectos de la problemática de la especie paleontológica: aplicación al caso de la diferenciación biométrica de Nassarius semistriatus (Brocchi, 1814) y N. elatus (Gould, 1845). Studia Geologica, 15, 7-36.

Fekih, M. 1975. Paleoecologie du Pliocene marin au Nord de la Tunisie. Annales des Mines et de la Geologie, 27, 1-193. Editions du Service geologique de Tunisie.

Ferrero E. e Merlino, B.1992. Ricostruzione paleoecologica 
di una malacofauna del bacino pliocenico Astigiano (Italia NW). Bolletino Malacologico, 28 (5-12), 101-138.

Gili, C. 1991. Els Nassariidae (Gastropoda: Prosobranchia) del Pliocè de la Mediterrània occidental. Tesi doctoral, Universitat de Barcelona, 1-563. Collecció Tesis Doctorals microfitxades núm. 1412 (1992). Publicacions de la Universitat de Barcelona.

Gili, C. 1992. Nassarius martinelli nov. sp. (Neogastropoda: Nassariidae) del Plioceno del Mediterráneo occidental. Revista Española de Paleontología, 7, 167-173.

Gili, C. y Martinell, J. 1990. Aportación al conocimiento del subgénero Sphaeronassa (Locard) (Neogastropoda, Nassariidae) del Plioceno del Mediterráneo y del Atlántico adyacente. Revista Española de Paleontología, 5, 19-33.

Gili, C. \& Martinell, J. 1992. A normative proposal for the figures of the protoconch of Gastropods. Bolletino Malacologico, 22 (5-12), 221-224.

Gili, C. \& Martinell, J. 1994. Relationship between species longevity and larval ecology in nassariid gastropods, Lethaia, 27, 291-299.

González Donoso, J.M. 1995. Algunas reflexiones sobre la problemática de los conceptos de especie. Revista Española de Paleontología, 10, 90-108.

Hoagland, K.E. \& Robertson, R. 1988. An assessment of poecilogony in marine invertebrates: phenomenon or fantasy? Biological Bulletin, 174, 109-125.

Jablonski, D. \& Lutz, R.A. 1980. Molluscan larval shell morphology: ecological and paleontological applications. In: Skeletal Growth of Aquatic Organisms (Eds.D.C. Rhoads and R.A. Lutz). Plenum, N.Y., 323 - 377.

Jablonski, D. \& Lutz, R.A. 1983. Larval ecology of marine invertebrates: paleobiological implications. Biological Review, 58, 21-89.

Koutsouveli, A. 1987. Étude stratigraphique des formations pliocènes et pleistocènes en Messenie occidentale (Peloponnese, Grece). Tesis Doctoral, Université d'AixMarseille II. 1-162.

Landau, B. 1984. A discussion of the molluscan fauna of two Pliocene localities in the provonce of Huelva (Spain), including descriptions of six new species. Tertiary Research, 6 (4), 135-155.

Le Roux, A. 1994. Sexualité et reproduction de Cyclope neritea (Mollusque gastéropode) dans le Golfe du Morbihan (Bretagne Sud). Haliotis, 23, 51-59.

Lecointre, G. 1952. Récherche sur le Néogène et le Quaternaire marins de la côte atlantique du Maroc, Vol 2. Paleontologie. Service Géologique du Maroc, Notices et Mémoires, 99, 1-173.

Malatesta, A. 1974. Malacofauna pliocenica umbra. Memorie Carta Geologica d'Italia, 13, 1- 498.

Martinell, J. 1982. Estudio de los Buccinacea (Neogastro- poda, Gastropoda) del Plioceno de l'Empordà (Catalunya). Descriptiva y sistemática. Butlletí de la Institució Catalana d'Història Natural, 48 (Sec. Geol. 3), 61-90.

Montefameglio, L., Pavia, G. e Rosa, D.A. 1979. Associazioni a molluschi del Tabaniano del Basso Monferrato (Alba, Italia). Bolletino Società Paleontologica Italiana, 18 (2), 173-199.

Oliverio, M. \& Tringali, L. 1992. Two sibling species of Nassariinae in the Mediterranean sea (Prosobranchia: Muricidae: Nassariinae). Bolletino Malacologico, 28 (512), 157-160.

Pallary, P. 1919. Le Ciclonassa neritea et ses derivations. Journal de Conchyliologie, 64 (1), 1-11.

Parenzan, P. 1970. Carta d'identità delle conchiglie del Mediterraneo. Vol I, Gasteropodi. Ed. Bios Taras, Taranto, 1-283.

Pavia, G. 1975. I molluschi del Pliocene inferiore di Monteu Roero (Alba, Italia NW). Bolletino Società Paleontologica Italiana, 14 (2), 1-175.

Pinna, G. e Spezia, L. 1978. Catalogo dei tipi del Museo Civico di Storia Naturale di Milano. Vol. I, Tipi dei Gasteropodi fossili. Atti della Società Italiana Scienze Naturaly, Museo Civico Storia Naturale di Milano, 119 (2), 125-180.

Sacco, F. 1904. I molluschi dei terreni terziari del Piemonte e d'ella Liguria. Parte 30, Clausen ed., Torino, 1-203.

Shuto, T. 1974. Larval ecology of prosobranch gastropods and its bearing on biogeography and paleontology. Lethaia, 7, 239-256.

Solsona, M. 1998. Paleobiologia dels Mesogasteròpodes del Pliocè del Mediterrani nord-occidental. Tesi doctoral. Universitat de Barcelona. 1-482.

Solsona, M. \& Martinell, J. (en prensa) Protoconch as a taxonomic tool in Gastropoda systematics. Application in the Pliocene Mediterranean Naticidae. Geobios.

Vera-Peláez, J.L., Muñiz, R., Lozano, M.C., Martinell, J., Domènech, R. y Guerra-Merchán, A. 1995a. Cancellaridae Gray, 1853 del Plioceno de la provincia de Málaga, España. Treballs del Museu de Geologia de Barcelona, 4, 133-179.

Vera-Peláez, J.L., Lozano, M.C., Muñiz, R., Gili, C., Martinell, J., Domènech, R., Palmqvist, P. y GuerraMerchán, A. 1995b. Estudio preliminar de la malacofauna del Plioceno de Estepona (Málaga, España). Iberus, 13 (2), 93-117.

Vermeij, G.J. 1971. Gastropod evolution and morphological diversity in relation to shell geometry. Journal of Zoology, 163, 15-23.

Zbyszewski, G. 1959. Étude structurale de l'aire Typhonique de Caldas da Rainha. Serviços Geológicos de Portugal, Mem. 3 (nova serie), 1-182. 